\title{
Enhancement of filamentation postcompression by astigmatic focusing
}

\author{
Benjamín Alonso, ${ }^{1, *}$ Rocío Borrego-Varillas, ${ }^{1}$ Íñigo J. Sola, ${ }^{1}$ Óscar Varela, ${ }^{2}$ Ayalid Villamarín, ${ }^{3}$ \\ M. Victoria Collados, ${ }^{3}$ Julio San Román, ${ }^{1}$ Juan M. Bueno, ${ }^{4}$ and Luis Roso ${ }^{2}$ \\ ${ }^{1}$ Grupo de Investigación en Óptica Extrema (GIOE), Universidad de Salamanca, Pl. Merced s/n, Salamanca E-37008, Spain \\ ${ }^{2}$ Centro de Láseres Pulsados, CLPU, Salamanca E-37008, Spain \\ ${ }^{3}$ Departamento de Física Aplicada, Universidad de Zaragoza, C/Pedro Cerbuna 12, Zaragoza, E-50009 Spain \\ ${ }^{4}$ Laboratorio de Óptica, Universidad de Murcia, Campus de Espinardo (CiOyN), Murcia E-30071, Spain \\ ${ }^{*}$ Corresponding author: b.alonso@usal.es
}

Received July 22, 2011; revised August 31, 2011; accepted September 3, 2011;

posted September 9, 2011 (Doc. ID 151517); published September 26, 2011

\begin{abstract}
The energy scaling up of pulse postcompression is still an open issue. In this work we analyze the use of astigmatic focusing to improve the output pulses in a filamentation based postcompression setup. Unlike spherical conditions, astigmatic focusing enhances the output energy and the spectral broadening of the filament. This is due to the increase of critical power, allowing a considerable improvement of the postcompression energy and stability in a simple way. We demonstrated compression from FWHM $100 \mathrm{fs}, 10 \mathrm{~nm}, 3 \mathrm{~mJ}$ input pulses to $13 \mathrm{fs}, 142 \mathrm{~nm}$, near $1 \mathrm{~mJ}$ pulses. (C) 2011 Optical Society of America

OCIS codes: $320.5520,190.7110$.
\end{abstract}

The process of filamentation consists of the self-guiding of high power laser pulses by the balance between the Kerr effect collapse trend and the ionization defocusing [1]. The range of applications is wide, including remote spectroscopy [2] and lightning control [3] among others. Filamentation stages have been successfully implemented for the postcompression of ultrashort laser pulses, taking advantage of the spectral broadening that occurs during the propagation [4], as an alternative to another widespread postcompression technique based on the self-phase modulation (SPM) of a beam in a gas filled hollow fiber [5]. The main constraint of these schemes originates from the upper limit for input and, consequently, output energies, which are not high enough for the requirements of the high power lasers available currently. Different solutions have been reported to overcome this issue. These include the use of planar waveguides [] $]$ or gas gradients inside the fiber $[7,8]$, and the use of circularly polarized [9] or chirped input pulses [10] in the case of filamentation. In the same way, molecular alignment was proposed to enhance the filament length and the supercontinuum generation [11], showing highenergy single filaments [12] and the compression of the pulses [13].

It has also been shown that both the spatial profile $[14,15]$ and phase $[16-18]$ of the laser beam fairly affect filamentation. In particular, adaptive control of the spatial phase provided single filamentation stabilization [17] and spatial homogenization [18]. Moreover, multifilamentation pattern has been controlled by inducing astigmatism (tilting a spherical lens) [19] and by using adaptive wavefront control [20] with a deformable mirror.

In this context, we further explore the effect of the laser beam spatial phase (i.e., the wavefront) in filamentation generation by introducing astigmatism with the focusing lens. The process of filamentation generation was studied in depth, as well as differences between astigmatic and nonastigmatic experimental conditions.

Results showed that astigmatic focusing enhanced the available output energy and the spectral broadening while keeping inside the single stable filamentation regime, allowing shorter and higher energy compressed pulses. This effect is thought to be caused by changes in the critical power due to nonsymmetric phase [21].

The experiments were performed using $100 \mathrm{fs}, 10 \mathrm{~nm}$ spectral FWHM, $10 \mathrm{~Hz}$ repetition rate, and $795 \mathrm{~nm}$ central wavelength input pulses, delivered by a Ti:Sapphire CPA laser system (Spectra Physics). In order to generate the filament, the laser beam was focused in both air and a cell filled with nitrogen gas. The gas cell was $2 \mathrm{~m}$ long and the lens was placed $20 \mathrm{~cm}$ before the entrance window of the cell to avoid effects at the entrance and exit windows. The effects of using astigmatic and spherical lenses were also compared. To optimize filamentation, the input energy, input beam diameter, and nitrogen pressure were used as control parameters, whereas the input chirp (near Fourier-transform limited) and linear polarization were fixed. Different parameters were characterized: energy, beam diameter, spectrum (Ava-Spec-2048, Avantes), output temporal profile (SPIDER from APE), and spatial profile (CCD Pulnix).

The filamentation process in air was firstly investigated by means of astigmatic ( 0.8 and $1 \mathrm{~m}$ nominal focal lengths) and spherical ( $1 \mathrm{~m}$ focal length) lenses. The input iris was fixed to $10 \mathrm{~mm}$ in diameter. The input energy was set to $4.8 \mathrm{~mJ}$ to get a stable single filament at the output. The images of the spatial profile evolution at different propagation distances from the astigmatic lens are shown in Fig. 1. The spatial profile variations as a function of the distance to the lens can be observed. From an elliptical vertical pattern, the profile changes to the first focus (along the vertical meridian). Going further it turns into the circle of least confusion, the second (horizontal) focus position, and, finally, an elliptical (divergent beam) with a circular filament in the center propagating nonlinearly. The experimental focus positions are slightly larger than the nominal values (although the same as those obtained from the linear regime calibration) since the lens was designed for a different wavelength than the one here used. 


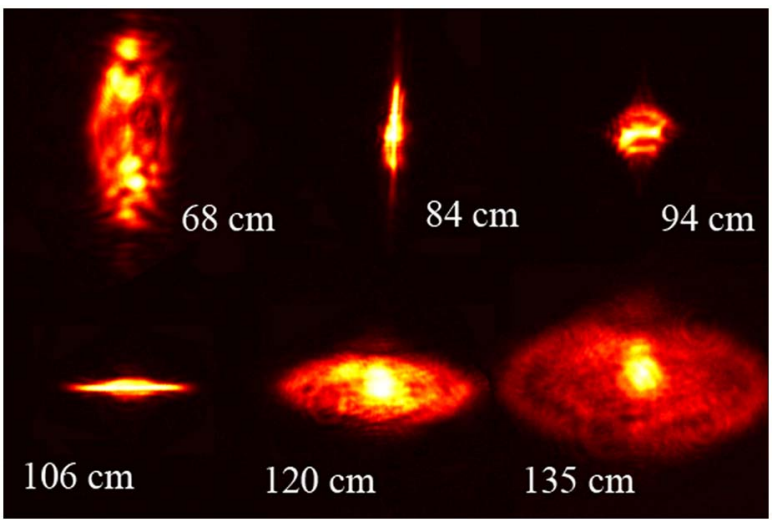

Fig. 1. (Color online) Spatial profile at different distances (inset) from the astigmatic lens in air (input energy: $4.8 \mathrm{~mJ}$ ).

In the second part of the experiment, the spatial profile was imaged as a function of the input energy (up to $6 \mathrm{~mJ}$ ) at a fixed distance from the lens $(144 \mathrm{~cm})$. Results are depicted in Fig. 2. For energy values lower than $1 \mathrm{~mJ}$ the pulse propagated linearly and the diffraction pattern due to the input iris is seen. As the input energy increased, it started to concentrate at the center $(2.4 \mathrm{~mJ})$ and a filament appeared (starting with $3.1 \mathrm{~mJ}$ and completed for $4.8 \mathrm{~mJ}$ ). Finally, multifilamentation was observed $(6.0 \mathrm{~mJ})$ along the vertical meridian (first focus direction), as expected [19]. The filament is then formed at the center of the transverse profile of the beam, and it does not inherit the elliptical shape of the linear part of the beam. Similar behavior was found for different astigmatic lenses. The suppression of multifilamentation has also been demonstrated by using molecular alignment [22]. The results in Figs. 1 and 2 show that the linear part of the beam propagates with the astigmatic input phase. On the other hand, the round profile of the filament preserved along the propagation suggests that the spatial quality of the filament is not affected by the astigmatic focusing, what supports the suitability for its use in a subsequent experiment. To verify this, we measured the wavefront of the output filament (inner core), obtaining the values of the Zernike coefficient for astigmatism at $0^{\circ}$ of $\mathrm{RMS}=-0.0021 \lambda$ (spherical) and $-0.0020 \lambda$ (astigmatic), respectively.

Once the mechanism had been studied, filamentation in similar conditions for a spherical $(f=1.0 \mathrm{~m})$ and two

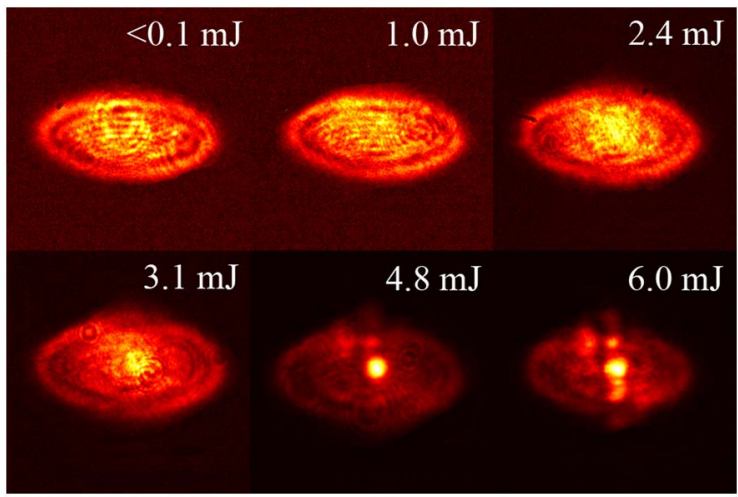

Fig. 2. (Color online) Output spatial profile for astigmatic focusing in air using different input energies (inset). The measurements were taken $144 \mathrm{~cm}$ after the lens. astigmatic lenses, labeled as Astig $1(f=0.8 \mathrm{~m}$ and $1.0 \mathrm{~m})$ and Astig2 $(f=1.0 \mathrm{~m}$ and $1.33 \mathrm{~m})$ were compared. For this aim a nitrogen gas cell at a pressure of 2104 mbar was used. The input iris was fixed to $5.6 \mathrm{~mm}$ diameter. The input/output energies (inner core selection for output energies) were $1.37 / 0.30 \mathrm{~mJ}, 1.71 / 0.55 \mathrm{~mJ}$, and $1.66 / 0.68 \mathrm{~mJ}$, for spherical, Astig1, and Astig2, respectively. These values confirm that energy transmission is suitable for applications when using astigmatic focusing. For the spherical lens the input energy was considerably reduced to avoid unstable filamentation as expected from our proposal for postcompression optimization.

Figure 3 compares the spectral density values of the input beam and those corresponding to the filament generated with spherical and astigmatic lenses. The spectral broadening obtained with the astigmatic lenses is considerably larger than the one obtained for the spherical lens, what allows shorter compressed pulses (Fouriertransform limits: 6.4, 4.3, and 4.4 fs for spherical, Astig1, and Astig2, respectively).

These results may be explained by the astigmatic beam propagation and the nonlinear nature of the filament. The increased Rayleigh length in astigmatic propagation due to two foci at different distances contributes to the spectral broadening due to SPM induced by Kerr effect during selfguiding. As reported in [16], longer focusing depths also lead to longer plasma channels. Moreover, noncircular beams [21,23] as well as nonsymmetric focusing [21] increase the critical power for self-focusing. This lengthening in the Rayleigh distance allows for the increase of the multifilamentation threshold, and therefore it is possible to get more stable and higher energy postcompression.

To understand this behavior, the Gaussian beam propagation for astigmatic and spherical cases in linear regime was simulated considering conditions close to the experiment $(6 \mathrm{~mm}$ input beam at intensity decrease to $\left.1 / e^{2}\right)$. The Rayleigh zone was calculated as the distance where the transverse area is below twice the minimum section (this is the confocal parameter, i.e., twice the Rayleigh length). As expected, for the astigmatic conditions the Rayleigh length was about twofold larger than that of the spherical case. The astigmatic focusing (two separated foci) was less pronounced and the peak intensity decreased by a factor 6 . This increase in Rayleigh length is equivalent to the result obtained when doubling the focal length of the spherical lens or using two stages [4] of filamentation. Notice that this statement affects the

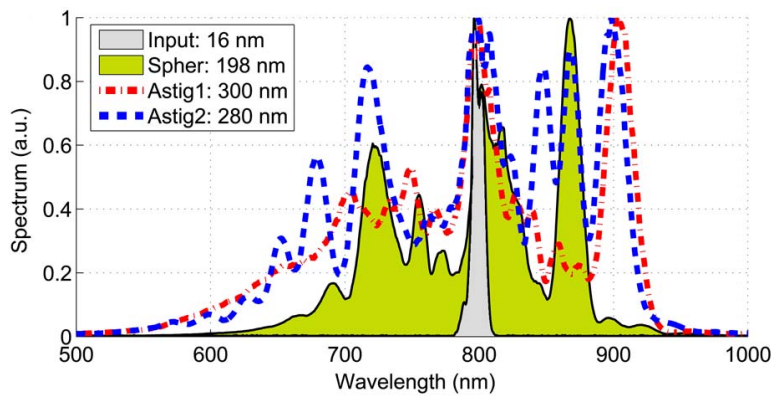

Fig. 3. (Color online) Normalized spectral density of the input pulse (gray filled), and output pulse for spherical (gold filled), Astig1 (red dash-dot), and Astig2 (blue dash) lenses. Measurements correspond to nitrogen gas filled cell at $P=2104$ mbar. Inset: $1 / e^{2}$ width. 

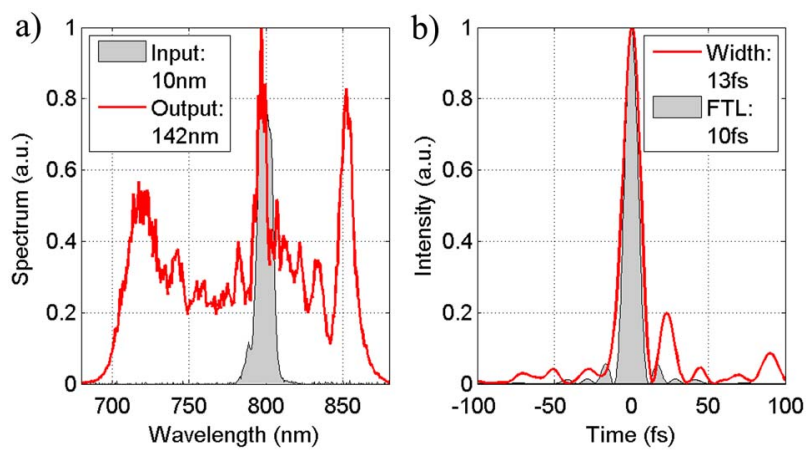

Fig. 4. (Color online) Optimized astigmatic postcompression in nitrogen gas ( $P=2570$ mbar): (a) Input (gray filled) and output (red solid line) spectrum. (b) Output intensity (red solid) and Fourier-transform limit of the corresponding spectrum (gray filled). Inset: respective FWHM.

spectral broadening enhancement, but not the increase of critical power and output energies.

Finally, we analyzed the benefits of using astigmatic lenses (instead of spherical) on pulse compression. The laser beam was focused by an astigmatic lens $(f=0.8$ and $1.0 \mathrm{~m})$ in nitrogen gas $(2570 \mathrm{mbar})$. Temporal measurements were limited by the range of the SPIDER ( $\geq 10 \mathrm{fs}$ ). Positive output spectral phase was compensated with a holographic-grating pair compressor before the SPIDER. Taking as starting point $100 \mathrm{fs}, 10 \mathrm{~nm}$ pulses, a broadband spectrum (FWHM $142 \mathrm{~nm}$ ) was produced [see Fig. 4(a)]. As shown in Fig. 4(b) the Fourier-transform limit of the spectrum was $10 \mathrm{fs}$ and the experimental pulse duration was $13 \mathrm{fs}$ [Fig. 4(b)]. In contrast, in stable regime and similar conditions of pressure, compressed pulses did not go below $17 \mathrm{fs}$ for the spherical lens. The input energy was $3 \mathrm{~mJ}$. The energy measured before the compressor was $1 \mathrm{~mJ}$, corresponding to the inner core selectionusing a $3 \mathrm{~mm}$ diameter iris-of the output filament. This energy transmission was similar to that obtained with spherical focusing (around 30\%). The energy of the compressed pulse was near $1 \mathrm{~mJ}$ due to the losses in the compressor. Moreover, the increase of critical power makes the filamentation more stable (keeping below the multifilamentation regime). This allows rising the input energy and thus also higher energy coupled in the output mode, in the millijoule level.

The particular focusing of astigmatic lenses led to a considerably larger (around twice) effective Rayleigh length. This implies that the laser beam nonlinear effects (filamentation) are achieved within longer distances producing a broader spectrum compatible with shorter pulses. The input energy can be increased thanks to the change of critical power, thus yielding higher output energies coupled in the filament mode.

The technique here presented is simple and does not require any nonconventional optical elements. Therefore, this technique is a suitable approach for higher energy postcompression experiments and can be used to enhance the output pulse duration shortening.
We acknowledge support from the Spanish Ministerio de Ciencia e Innovación through the Consolider Program SAUUL (CSD2007-00013), Research project FIS200909522, and grant programs Formación de Profesorado Universitario (for B. Alonso and R. Borrego-Varillas) and Ramón y Cajal (for I. J. Sola), and from the Junta de Castilla y León through the Program for Groups of Excellence (GR27). We also acknowledge support from the Centro de Láseres Pulsados, Salamanca, Spain.

\section{References}

1. A. Braun, G. Korn, X. Liu, D. Du, J. Squier, and G. Mourou, Opt. Lett. 20, 73 (1995).

2. K. Stelmaszczyk, P. Rohwetter, G. Méjean, J. Yu, E. Salmon, J. Kasparian, R. Ackermann, J.-P. Wolf, and L. Wöste, Appl. Phys. Lett. 85, 3977 (2004).

3. J. Kasparian, R. Ackermann, Y.-B. André, G. Méchain, G. Méjean, B. Prade, P. Rohwetter, E. Salmon, K. Stelmaszczyk, J. Yu, A. Mysyrowicz, R. Sauerbrey, L. Wöste, and J.-P. Wolf, Opt. Express 16, 5757 (2008).

4. C. P. Hauri, W. Kornelis, F. W. Helbing, A. Heinrich, A. Couairon, A. Mysyrowicz, J. Biegert, and U. Keller, Appl. Phys. B 79, 673 (2004).

5. M. Nisoli, S. De Silvestri, O. Svelto, R. Szipöcs, K. Ferencz, Ch. Spielmann, S. Sartania, and F. Krausz, Opt. Lett. 22, 522 (1997).

6. A. Suda, M. Hatayama, K. Nagasaka, and K. Midorikawa, Appl. Phys. Lett. 86, 111116 (2005).

7. J. Chen, A. Suda, E. J. Takahashi, M. Nurhuda, and K. Midorikawa, Opt. Lett. 33, 2992 (2008).

8. S. Akturk, C. L. Arnold, B. Zhou, and A. Mysyrowicz, Opt. Lett. 34, 1462 (2009).

9. O. Varela, A. Zaïr, J. San Román, B. Alonso, I. J. Sola, C. Prieto, and L. Roso, Opt. Express 17, 3630 (2009).

10. B. Alonso, O. Varela, I. J. Sola, J. San Román, A. Zaïr, C. Méndez, and L. Roso, Appl. Phys. B 101, 15 (2010).

11. J. Wu, H. Cai, H. Zeng, and A. Couairon, Opt. Lett. 33, 2593 (2008).

12. H. Cai, J. Wu, X. Bai, H. Pan, and H. Zeng, Opt. Lett. 35, 49 (2010).

13. J. Liu, Y. Feng, H. Li, P. Lu, H. Pan, Jian Wu, and H. Zeng, Opt. Express 19, 40 (2011).

14. T. D. Grow and A. L. Gaeta, Opt. Express 13, 4594 (2005).

15. D. Majus, V. Jukna, G. Valiulis, and A. Dubietis, Phys. Rev. A 79, 033843 (2009).

16. Q. Sun, H. Jiang, Y. Liu, Y. Zhou, H. Yang, and Q. Gong, J. Opt. A 7, 655 (2005).

17. T. Pfeifer, L. Gallmann, M. J. Abel, D. M. Neumark, and S. Leone, Opt. Lett. 31, 2326 (2006).

18. D. Walter, S. Eyring, J. Lohbreier, R. Spitzenpfeil, and C. Spielmann, Appl. Phys. B 88, 175 (2007).

19. G. Fibich, S. Eisenmann, B. Ilan, and A. Zigler, Opt. Lett. 29, 1772 (2004).

20. C. P. Hauri, J. Gautier, A. Trisorio, E. Papalazarou, and P. Zeitoun, Appl. Phys. B 90, 391 (2008).

21. C. R. Giuliano, J. H. Marburger, and A. Yariv, Appl. Phys. Lett. 21, 58 (1972).

22. J. Wu, H. Cai, Y. Peng, Y. Tong, A. Couarion, and H. Zeng, Laser Phys. 19, 1759 (2009).

23. G. Fibich and B. Ilan, J. Opt. Soc. Am. B 17, 1749 (2000). 